\title{
Thermal Instability
}

\author{
Dr Amna Mohammed Osman Elzebair
}

Faculty of: Art and Since-Mathematical department, University of Qassim - Branch of Al-Asyah, Kingdom of Saudi Arabia

\begin{abstract}
This paper discusses the thermal instability when a fluid is heated form below. The classic example of this, is a horizontal layer of fluid with its lower side hotter than its upper. The basic state is then one of rest with light fluid below heavy fluid. When the temperature difference across the layer is great enough, the stabilizing effects of viscosity and thermal conductivity are overcome by the destabilizing buoyancy, and an overturning instability ensues as thermal convection and an overturning instability ensues as thermal convection.
\end{abstract}

Keywords: Instability - Thermal conductivity-Bènard convection - Raleigh number -Navier-Stockes equations

\section{Introduction}

Bènard worked with very thin layers, only about $1 \mathrm{~mm}$ deep, standing on a levelled metallic plate which was maintained at a uniform temperature. The upper surface was usually free, and being in contact with the air was at a lower temperature. Various liquids were employed-some, indeed, which would be solids under ordinary conditions. The layer rapidly resolves itself into a number of cells, the motion being an ascension in the middle of a cell and a descension at the common boundary between a cell and its neighbours... . The cells acquire surfaces nearly identical, their forms being nearly regular convex polygons of, in general, 4 to 7 sides. The boundaries are vertical.... Fig.2.1 shows a plan of the convection cells in a silicone oil, with regular hexagons as the predominantpolygons.

Stimulate by Bènard’s experiments, Rayleigh (1916a) formulated the theory of convective instability of a layer of fluidon and boundary conditions to model the experiments, and derived the linear equation for normal modes. between horizontal planes. He chose equations of motion he then showed that instability would occur only when the adverse temperature gradient was so large that the dimensionless parameter $g \alpha \beta d^{4} / \kappa v$ exceeded a certain critical value - Here $g$ is the acceleration due to gravity, $\boldsymbol{a}$ the coefficient of thermal expansion of the fluid, $b=-d \mathrm{Q} / d z$ the magnitude of the vertical temperature gradient of the basic state of rest, $d$ the depth of the layer of the fluid, $\boldsymbol{\kappa}$ its thermal diffusivity and $\boldsymbol{n}$ its kinematic viscosity. This parameter is now called the Raleigh number. We shall denote it by $\mathbf{R a}$ in this paper, $R a$. The Raleigh number is characteristic ratio of the destabilizing effect of buoyancy to the stabilizing effects of diffusion and dissipation.

\section{Thermal Instability}

\subsection{Equations of Motion}

\subsubsection{The Exact Equations}

The equations of motion of a heat-conducting viscous fluid under the action of gravity can be found in textbook (e.g. Batchelor 1967). In the notation of Cartesian tensors with position vector $\mathbf{x}=\mathrm{x}_{\mathrm{j}}$ and velocity $\mathbf{u}=\mathrm{u}_{\mathrm{j}}(\mathrm{j}=1,2,3)$, the equations are as follows. The equation of continuity is

$$
\frac{\Phi r}{\Phi t}+\frac{\pi\left(r u_{j}\right)}{\Phi x_{j}}=0
$$

The equations of motion are the Navier-Stockes equations,

$$
\rho \frac{D u_{i}}{D t}=-g \rho \delta_{i 3}+\frac{\partial \sigma_{i j}}{\partial x_{j}},
$$

where $D / D t=\partial / \partial t+u \cdot \nabla$, the $x_{3}$-axis is the upward vertical, the stress tensor is given by

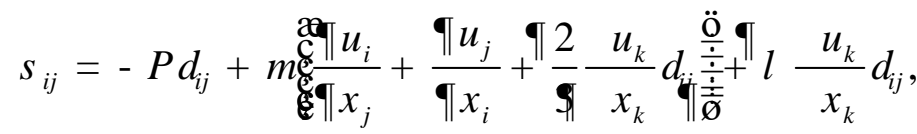

$m$ is the coefficient of dynamic viscosity of the fluid, and

l is that of bulk viscosity ( or secondviscosity). The equation of energy, or of heat conduction, is

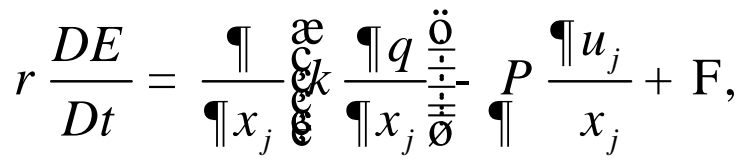




\section{International Journal of Science and Research (IJSR) \\ ISSN (Online): 2319-7064}

Index Copernicus Value (2013): 6.14 | Impact Factor (2014): 5.611

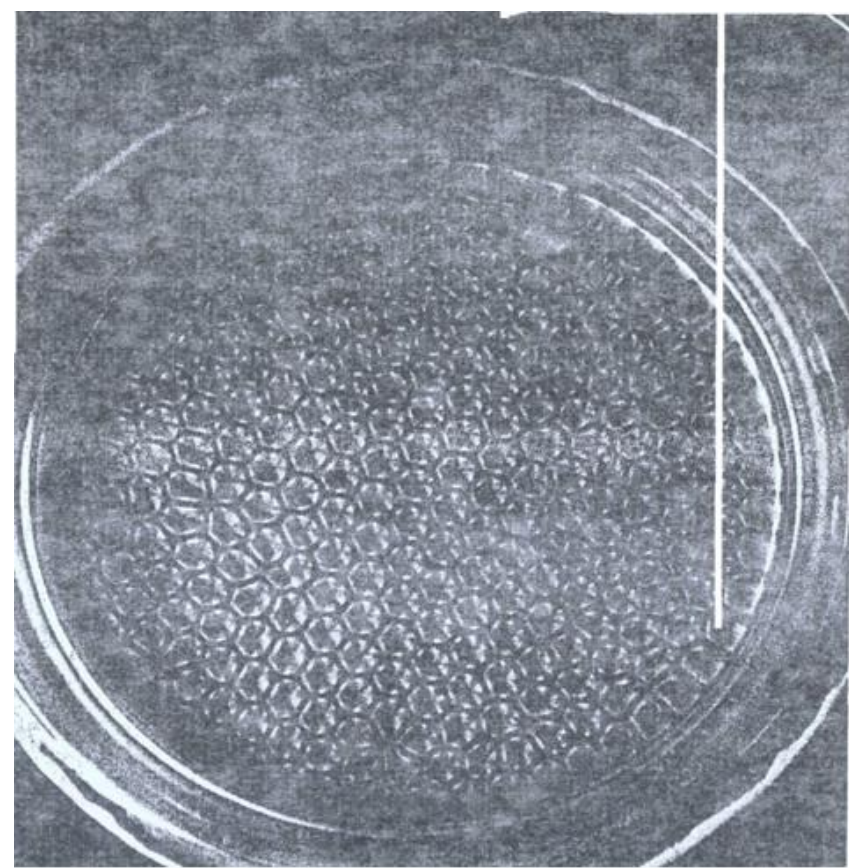

Figure 2.1.1: Be'nard cells under an air surface ( from Koschmieder\&pallas 1974)

where $E$ is the internal energy per unit mass of the fluid, $k$ is the thermal conductivity, $q$ is the temperature, and the rate of viscous dissipation per unit volume of fluid is given by

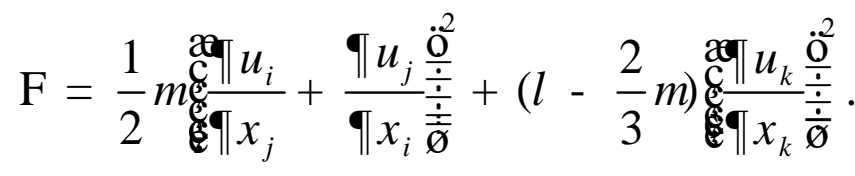

For a calorically perfect gas $E=c_{V} q$, and for a liquid $E=c q$, where $C_{n}$ is the specific heat at constant volume and $c$ the specific heat.

In general, the equations of state for a fluid specify $\boldsymbol{P}, \boldsymbol{\mu}, \boldsymbol{\lambda}, \boldsymbol{k}, \boldsymbol{C}$ and Eas functions of $\mathbf{P}$ and $q$. For layers of real fluid in which the pressure does not vary much, these functions are almost independent of $\mathbf{P}$.

\section{The Boussinesq approximation}

To these equations of motion, Rayleigh (1916a) applied theBoussinesq approximation, due independently to Oberbeck (1879) and Boussinesq(1903). The basis of this approximation is that there are flows in which the temperature varies little, and therefore the density varies little, yet in which the buoyancy drives the motion. Then the variation of density is neglected everywhere except in the buoyancy. On the basis of this approximation for small temperature difference between the bottom and top of the layer of fluid,

$$
r=r_{o}\left\{1-a\left(q-q_{o}\right)\right\},
$$

where $r_{o}$ is the density of the fluid at the temperature $q_{o}$ of the bottom of the layer and $\boldsymbol{Q}$ is the constant coefficient of cubical expansion. For a perfect gas, $a=1 / q_{0} / 3^{\prime} 10^{-3} K^{-1}$, and for a typical liquid used in experiments $a \gg 5^{\prime} 10^{-4} K^{-1}$. If $q_{o}-q$ \ $10 K$, then $\left(r-r_{o}\right) / r_{o}=a\left(q_{o}-q\right)=1$, but nevertheless the buoyancy $g\left(\rho-\rho_{0}\right)$ is of the same order of magnitude as the inertia, acceleration or viscous stresses of the fluid and so is not negligible. For most real fluids $d \mu / \mu d \theta, d k / k d \theta, d c / c d \theta^{\wedge} \alpha$, so that $m, k$ and $c$, or $C_{V}$, are treated as constants in the Boussinesq approximation. (The coefficient of bulk viscosity $l$ is neglected, because it only arises as a factor of $\llbracket u_{j} / \mid x_{j}$, which is of order $\boldsymbol{a}$. In short, one approximates the thermodynamic variables as constants except for the pressure and temperature and except for the density when multiplied by $g$. This approximation works well for flows with temperature differences of a few degrees or less, such as those in Bènardexperiments, and can be formally justified by dimensional analysis (Spiegel \&Veronis 1960, Mihaljan 1962). Here we shall give only a partial justification.

The differences of density in the continuity equation(2.2.1) are of order $\boldsymbol{a}$, so the approximation gives

$$
\frac{\Phi u_{j}}{\prod x_{j}}=0
$$

as for an incompressible fluid. Then the stress tensor is given by

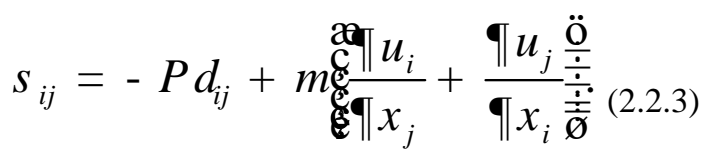

Again, on treating $r$ and $\boldsymbol{\mu}$ as constants in each term other than the buoyancy, the Navier-Stockes equations become

$$
\frac{D u_{i}}{D t}=-\frac{\partial}{\partial x_{i}}\left(\frac{p}{\rho_{0}}+g z\right)-\alpha g\left(\theta_{0}-\theta\right) \delta_{i 3}+v \Delta u_{i},
$$

where the Laplacian operator is given by $\mathrm{D}=\boldsymbol{q}^{2} / \boldsymbol{\Phi} x_{j}^{2}$.

Next we must simplify the heat equation (1.1.4). Firstly, note that, if $V$ is representative velocity scale of the flow, $d$ a length scale, and $q_{o}-q_{1}$ a scale of temperature difference, then the ratio of the rate of production of heat by internal friction to the rate of transfer of heat is

$$
\mathrm{F} / r \frac{D(c q)}{D t} » m V^{2} d^{-2} / r_{0} c\left(q_{0}-q_{1}\right) V d^{-1}=n V / c\left(q_{0}-q_{1}\right) d \text {, }
$$

$q_{1}$ being the temperature of the top of the layer of thickness $d$. Now, for a typical gas $n / c_{V} \gg 10^{-8} s K$ and for typical liquid $n / c$ » $10^{-9} s K$, which shows that the ratio is very 


\section{International Journal of Science and Research (IJSR)

small forboth gases and liquids unless $V /\left(q_{0}-q_{1}\right) d$ is very large.

Therefore we shall neglect $\Phi$. Secondly, note that the heating due to compression is

$$
-P \frac{\| u_{j}}{\llbracket x_{j}}=\frac{P}{r} \frac{D r}{D t}=a P \frac{D q}{D t}
$$

For a perfect gas, $P=\left(c_{p}-c_{v}\right) r q$ and $a=1 / q$ Therefore

$$
r \frac{D E}{D t}+P \frac{\| u_{j}}{\mathbb{\top} x_{j}} @ c_{p} r \frac{D q}{D t},
$$

and the heating due to compression is not negligible in comparison to the heat transfer, as approximation (2.2.2) might have led one to expect. For liquids, however, the heating is negligible at normal pressure. The reason for this difference between gases and liquids is chiefly because, although the heating due to compression is typically only an order of magnitude less for a liquid than for a gas, the heat transfer is proportional to the density of the fluid, and a typical liquid is $10^{3}$ times more dense than a typical gas. With all of these approximations, the heat equation becomes

$$
\frac{D q}{D t}=k \mathrm{D} q,
$$

where the thermal diffusivity $\kappa=k / \rho_{0} c_{p}$ for perfect gas and $k / \rho_{0} c$ for a liquid. Equations (2.2.3), (2.2.5) and (2.2.6) are called the Boussinesq equations and describe the motion of Boussinesqfluid.

\section{The Stability Problem}

\subsection{The Linearized Equations}

Rayleigh (1916a) modelled Bènard's experiments as the instability of Boussinesq fluid at rest between two infinite horizontal planes at different temperatures. Let the planes have equations $Z_{*}=0$ and $d$, where the temperatures are $q_{0}$ and $q_{1}$ respectively. Here we denote a dimensional variable by subscripted asterisk to prepare for our choice of dimensionless variables; e.g. we shall soon take $z=z_{*} / d$ to be the dimensionless variable of height. Then the equations of motion give the basic state with

$$
U_{*}=0, \mathrm{Q}_{*}=q_{0}-b Z_{*}
$$

$p_{*=} p_{0}-g \rho_{0}\left(z_{*}+\frac{1}{2} \alpha \beta z_{*}^{2}\right)$ for $0 £ z_{*} £ d$, where the basic temperature gradient $b=\left(q_{0}-q_{1}\right) d$. We anticipate that there can be instability only when $q_{0}>q_{1}$, i.e. when there is an adverse temperature gradient and $b \mathrm{f}$.

$$
\mathbf{u}_{*}=\mathbf{u}^{\prime} *\left(\mathbf{X}_{*}, \mathrm{t}\right),
$$

$q_{*}=\mathrm{Q}_{*}\left(z_{*}\right)+q_{*} \phi\left(\mathbf{x}_{*}, t_{*}\right), P_{*}=P_{*}\left(z_{*}\right)+P_{*} \phi\left(\mathbf{x}_{*}, t_{*}\right)$, and linearizing the Boussinesq equations for small perturbations $\mathbf{u} \underset{*}{\notin}, q_{*} \notin, P_{*} \notin$, it follows that

$$
\tilde{\mathrm{N}}_{*} . \mathbf{u} \notin=0
$$

$$
\begin{aligned}
& \frac{\boldsymbol{\Phi} \mathbf{u} \phi}{\mathbb{\Upsilon} t_{*}}=-\frac{1}{r_{0}} \tilde{\mathrm{N}}_{*} P_{*} \phi+a \mathbf{g} q_{*} \phi \mathbf{k}+n \mathrm{D}_{*} \mathbf{u}_{*} \phi \\
& \frac{\prod q *}{\| t_{*}}-b \mathrm{w}_{*} \phi=k \mathrm{D}_{*} q_{*} \phi
\end{aligned}
$$

In the absence of any basic velocity, we seek convection driven by buoyancy and moderated by viscosity and thermal diffusivity, so it is convenient to use scales $d$ of length, $d^{2} / k$ of time, and $b d=q_{0}-q_{1}$ of temperature difference. (One may equivalently use $d^{2} / n$ as the time scale; this somewhat simpler if $n$ ? $k$.) Accordingly we define the dimensionless variables

$$
\begin{gathered}
\mathbf{x}=\mathbf{x}_{*} / d, t=k t_{*} / d^{2}, \mathbf{u}=d \mathbf{u}^{\prime} * / K \\
q=q_{*} \notin / b d, P=d^{2} P_{*}^{\prime} / r_{0} k^{2} .
\end{gathered}
$$

Then the linearized stability equations (3. 1.2)- (3. 1.4) become

$$
\begin{array}{r}
\tilde{N} . \mathbf{u}=0, \\
\frac{\Phi \mathbf{u}}{\Phi t}=-\tilde{\mathrm{N}} P+R \operatorname{Pr} q \mathbf{k}+\operatorname{Pr} \mathrm{D} \mathbf{u}, \\
\frac{\prod q}{\mathrm{q} t}-\mathrm{w}=\mathrm{D} q,
\end{array}
$$

respectively, where the dimensionless Rayleigh number is given by

$$
R=g a b d^{4} / k n
$$

and the Prandtl number by

$$
\operatorname{Pr}=n / k \text {. }
$$

Note that the Rayleigh number is positive when the lower boundary is the hotter one ( $\left.q_{0}>q_{1}\right)$ and is seen to be a characteristic ratio of the buoyancy to the viscous forces. Also note that the Prandtl number is an intrinsic property of the fluid, not of the flow; it measures the ratio of the rates of molecular diffusion of momentum and heat. We can now easily eliminate all the dependent variables except $w$, to get a single stability equation. The curl of equation (3. 1.7) gives

$$
\frac{\prod \omega}{\mathbb{\Upsilon} t}=R \operatorname{Pr}\left(\tilde{\mathrm{N}} q^{\prime} \mathbf{k}\right)+\operatorname{PrD} \omega
$$

where the velocity $\omega=\tilde{N}^{\prime} \mathbf{u}$. The curl of equation (3.1.11) in turn gives, after use of equation (2.1.6),

On putting 


\section{International Journal of Science and Research (IJSR) \\ ISSN (Online): 2319-7064}

Index Copernicus Value (2013): 6.14 | Impact Factor (2014): 5.611

In particular ,

$$
\frac{\Phi}{\llbracket t} \mathrm{D} \mathbf{u}=R \operatorname{Pr}\left(\mathrm{D} q \mathbf{k}-\tilde{\mathrm{N}} \frac{\boldsymbol{\Phi} q}{\llbracket z}\right)+\operatorname{Pr}^{2} \mathbf{u} .
$$

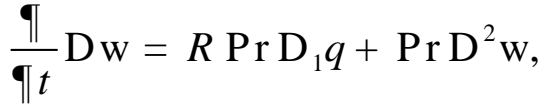

where the horizontal Laplacian $\mathrm{D}_{1}=\boldsymbol{q}^{2} / \boldsymbol{q} x^{2}+\mathbb{q}^{2} / \mathbb{q} y^{2}$. Finally elimination of $\theta$ from equations (3.1.8),(3.1.13) gives

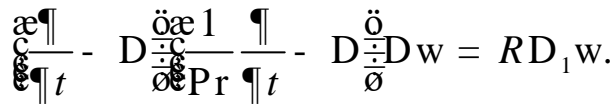

Similarly it can be shown that $\theta$ satisfies the same equation.

It can be shown from the equation of continuity that

$$
\begin{aligned}
& \mathrm{D}_{1} u=-\frac{\boldsymbol{\Phi}^{2} \mathrm{w}}{\llbracket x \| z}-\frac{\llbracket w_{3}}{\llbracket y}, \\
& \mathrm{D}_{1} v=-\frac{\mathbb{\|}^{2} \mathrm{w}}{\llbracket y \| z}+\frac{\llbracket w_{3}}{\llbracket x},
\end{aligned}
$$

where $w_{3}=\llbracket v / \rrbracket x-\rrbracket u / \rrbracket y$ is the vertical component of vorticity.

This is given by the vertical component of equation (3. 1.11), namely

$$
\frac{\llbracket w_{3}}{\llbracket t}=\operatorname{PrD} w_{3}
$$

So $u, v$ can be found by solving the Poisson equations (3. 1.15), (3. 1.16) when $\mathrm{W}$ has been found by solving equation (3.1.14) and $w_{3}$ by solving the diffusion equation (3.1.17).

\section{References}

[1] Amano, H. Inoue, T.A New PSS Parameter Design Using NonlinearStability AnalysisPower Engineering Society General Meeting, 2007. IEEEDigital Object Identifier: 10.1109/PES.2007.385708Publication Year: 2007 , Page(s): 1 - 8 IEEE Conferences

[2] Arnold, V. I. (1965a).Conditions for nonlinear stability of stationary plane curvilinear flows of an ideal fluid .Dokl.Akad. Nauk SSSR 162,975-8. Translated in Soviet Math. Dokl. 6, 773-7(1965).[pp.432,434.]

[3] Arnold, V. I. (1991), Mathematical Methods of Classical Mechanics, 2nd Ed., Springer

[4] Batchelor, G.K. (1967). An introduction to fluid DynamicsCambridgeUniversity press.

[5] Batchelor, T.B. (1962).T. fluidMech.14,593. Cambridge University press.

[6] Bénard, H (1900). Les tourbillons cellulairesdansune nappe liquide. Revue G én. Sci. Pur. Appl.11, 1261-71 and 1309-28. [p. 32.]

[7] Benjamin, T. B. (1961). The development of threedimensional disturbances in an unstable film of liquid flowing down an inclined plane. J. Fluid Mech.10,40119.
[8] Benjamin, T. B. (1972). The stability of solitary waves, Proc. Roy. Soc, A 328, 153-83.

[9] Boussinesq, J. (1903) . Théorieanalytique due la chaleur, vol.2, p. 172. Paris: Gauthier-Villars. [p. 35.]

[10] chandrasekhar, S. (1954b). The stability of viscous flow between rotating cylinders. Mathematika 1, 5-13.[p. 99.]

[11] chandrasekhar,S. (1961).Hydrodynamic and hydromagnetic stability . Oxford: Clarendon Press.[pp. 25,28.49,62,64,66,67,79,93,98,99,103,110,121,205,327,3 40.]

[12] Couette, M.(1890). Études sur le frottement des liquids. Ann. Chim. Phys. (6) 21,433-510.[p. 70.]

[13] Cole, J.A. (1976). Taylor-vortex instability and annuluslength effects. J. fluid Mech. 75,1-15.[p. 449.]

[14]Colson, D.(1954). Wave-cloud formation at Denever.Weather-wise 7 , 34-5. [p. 22.] 